\title{
ROBOT KINEMATIC CALIBRATION ACCURACY USING GENETIC ALGORITHM
}

\author{
Salem Samak \\ Prod. Eng. \& Mech. Design Dept. \\ Faculty of Engineering \\ Menoufia University \\ Shebin El-Kom, EGYPT
}

\begin{abstract}
Position and orientation accuracy of the end-effector is influenced by the precision of kinematic parameters elements of the robot. Thus, good precision requires good knowledge of robot physical parameters values. Howevef, this condition can be difficult to meet in practice. Hence, calibration techniques can be devised in order to improve the robot accuracy through estimation of those particular parameters. In this paper, the Genetic Algorithm (GA) is used to calibrate the robot kinematic accuracy. A kinematic model is formulated and conducted as an optimization problem for serial robot manipulators. The objective is to analyze and evaluate the performance of the GA in optimizing such robot kinematic accuracy. In this algorithm, the errors in the robot parameters represent the parents and offspring population and the error matrix norms represent the cost functions. The convergence and effectiveness of the presented model are demonstrated by a numerical example.
\end{abstract}

\section{KEYWORDS}

Manipulators, Robotics, Calibration, Accuracy, Genetic Algorithm.

\section{INTRODUCTION}

Genetic Algorithm (GA) [1] is a rather new search tool in robotics, which exhibits high efficiency in certain multi-modal and multi-dimensional domains. The algorithm has been implemented to handle the optimization problems for a number of different application areas, such as robotics [2], composite materials

Manuscript received from Dr. SALEM SAMAK

Accepted on : 28/9/2000

on : $6 / 9 / 2000$

Engineering Research Bulletin, Vol 23,No 4, 2000 Minufiya University, Faculty of

Engineering, Shebien El-Kom, Egypt, ISSN 1110-1180 
design, scheduling [3] and also salesman problems [4]. Recently, it is a gate for the automatic design, which is the best way to develop artificial intelligence. It can be easily adapted to the planning problems for many types of assembly machines [5]. The GA uses population as parents to represent possible solutions. The fitness of all of the individuals in the population is evaluated by its cost. The genetic operators (such as crossover, inversion, rotation and mutation) are applied to generate new population called offspring in an iterative procedure to obtain nearly optimal solutions.

The position and orientation of the end-effector would have no errors other than those caused by imperfection of the repeatability and dynamic effects. However, in more sophisticated applications, errors in the position and orientation of the end-effector result from the kinematic parameters errors as well, which are mostly due to manufacturing and/or measurement errors.

The calibration of robot manipulators has attracted many researchers. Veitschegger and $\mathrm{Wu}[6]$ have presented a result comparison between two models. The first model has ignored the higher order terms and did not address the special case of two consecutive parallel joints, while the second model has considered both cases. Wu [7] has used a new technique to correct the kinematic errors of robot manipulator. Vukobratovic and Borovac [8] have investigated the influence of the deviations of the links nominal measures (due to manufacturing tolerances) on the accuracy of positioning the manipulator tip for various mechanism configurations. Bruyninckx et al. [9] have developed a systematic and fully general model-based approach to compliant robot motion, taking into account uncertainties in the geometry of the manipulated object and the environment with which it is in contact. Samak et al. [10] have studied the effect of kinematic perturbations on robot precision. Kazerounian and Qian [11] have presented a kinematic calibration model for position and orientation of serial manipulator end-effector errors due to repeatability imperfections.

The present paper introduces a new perspective proposal in order to analysis, implement and evaluate the performance of the GA in optimizing the robot kinematic accuracy. The kinematic relationships have been described by using the zero-reference-position (ZRP) method. The prescribed analysis showed improvement in the robot accuracy precision.

\section{ZERO POSITION ANALYSIS METHOD}

The zero position analysis method was introduced by Gupta [12]. It has the advantages that it is not prone to the discontinuity difficulties as those in the Denavit Hartenberg notation. Due to the nature of this method, small changes in the structure inherently correspond to small changes in the structure parameters. It has also proven its effectiveness and versatility in many works on both kinematic and dynamic analysis of robot manipulators [13]. The joint coordinate systems in this method are not used. Instead, a convenient reference position of 
the robot is chosen and the following vectors are defined in the world coordinate system,

$\boldsymbol{u}_{0 i} \Rightarrow \mathrm{a}$ unit vector along joint axis $\mathrm{i}$.

$\boldsymbol{b}_{0 i} \Rightarrow$ a body vector which connects a point on joint (i-1) to a point on joint $\mathrm{i}$.

$\boldsymbol{u}_{0 a}$ and $\boldsymbol{u}_{0 t} \Rightarrow$ two perpendicular vectors fixed on the end-effector.

All the above-mentioned parameters are given in their ZRP (with zero subscript). They are converted to the current position as the manipulator moves to new positions. The current vector are derived from their ZRP vectors as follows,

$$
\begin{gathered}
\boldsymbol{u}_{\boldsymbol{i}}=\boldsymbol{R}_{\boldsymbol{i}} \boldsymbol{u}_{0 i} \\
\boldsymbol{b}_{i+1}=\boldsymbol{R}_{i} \boldsymbol{b}_{0, i+1} \\
\boldsymbol{u}_{a}=\boldsymbol{R}_{\boldsymbol{h}} \boldsymbol{u}_{0 a} \text { and } \boldsymbol{u}_{\boldsymbol{t}}=\boldsymbol{R}_{\boldsymbol{h}} \boldsymbol{u}_{0 t}
\end{gathered}
$$

Where $\mathrm{i}=1,2, \ldots, \mathrm{n}$; the 3 by 3 rotation matrix $\mathbf{R}_{\mathrm{i}}$, for a revolute joint, is defined as

$$
\boldsymbol{R}_{i}=\boldsymbol{R}\left(q_{1}, u_{o l}\right) \boldsymbol{R}\left(q_{2}, u_{o 2}\right) \quad \cdots \quad R\left(q_{i}, u_{o i}\right)=\prod_{j=l}^{i} R\left(q_{j}, u_{o j}\right)
$$

Hence, for n-revolute joints manipulators, the above equation represents the hand orientations $\boldsymbol{R}_{\mathrm{h}}$ as

$$
R_{h}=\prod_{i=1}^{n} R\left(q_{i}, u_{0 i}\right)
$$

The matrix $\boldsymbol{R}\left(q_{i}, \boldsymbol{u}_{\boldsymbol{i}}\right)$ represents a rotation by $q_{i}$ about a screw axis $\boldsymbol{u}_{o i}$. It can be written as [14].

$$
R\left(q_{i}, u_{o i}\right)=\left(\begin{array}{ccc}
\left(u_{x}^{2}-l\right) V_{i}+l & u_{x} u_{y} V_{i}-u_{z} S_{i} & u_{x} u_{z} V_{i}+u_{y} S_{i} \\
u_{x} u_{y} V_{i}+u_{z} S_{i} & \left(u_{y}^{2}-I\right) V_{i}+I & u_{y} u_{z} V_{i}-u_{x} S_{i} \\
u_{x} u_{z} V_{i}-u_{y} S_{i} & u_{y} u_{z} V_{i}+u_{x} S_{i} & \left(u_{z}^{2}-l\right) V_{i}+1
\end{array}\right)
$$

where, $V_{i}=1-\cos \left(q_{i}\right)$ and $S_{i}=\sin \left(q_{i}\right)$, and $u_{x}, u_{y}$ and $u_{z}$ are components of the unit vector $\boldsymbol{u}_{0 i}$. If the $\mathrm{i}^{\text {th }}$ joint is prismatic, then $\boldsymbol{R}\left(q_{i}, \boldsymbol{u}_{o i}\right)$ is replaced with a 3 by 3 identity matrix.

Equation (6) can be decomposed as follows,

$$
R\left(q_{i}, u_{o i}\right)=V_{i} A+S_{i} B+I
$$

where 


$$
A=\left(\begin{array}{lll}
u_{x}^{2}-1 & u_{x} u_{y} & u_{x} u_{z} \\
u_{x} u_{y} & u_{y}^{2}-1 & u_{y} u_{z} \\
u_{x} u_{z} & u_{y} u_{z} & u_{z}^{2}-l
\end{array}\right) \quad \text { and } \quad B=\left(\begin{array}{ccc}
0 & -u_{z} & u_{y} \\
u_{z} & 0 & -u_{x} \\
-u_{y} & u_{x} & 0
\end{array}\right)
$$

\section{ROTATIONAL ERROR MODEL}

For small changes in $u_{x}, u_{y}$ and $u_{z}$, the corresponding small change in the rotation matrix $R\left(q_{i}, u_{o i}\right)$ can be shown to be

$$
\delta R\left(q_{i}, u_{o i}\right)=R_{x i} \delta u_{0 i, x}+R_{y i} \delta u_{0 i, y}+R_{z i} \delta u_{0 i, z}
$$

where

$$
\boldsymbol{R}_{x i}=\left(\begin{array}{ccc}
2 X_{i} & Y_{i} & Z_{i} \\
Y_{i} & 0 & -S_{i} \\
Z_{i} & S_{i} & 0
\end{array}\right), \boldsymbol{R}_{y i}=\left(\begin{array}{ccc}
0 & X_{i} & S_{i} \\
X_{i} & 2 Y_{i} & Z_{i} \\
-S_{i} & Z_{i} & 0
\end{array}\right) \text { and } \boldsymbol{R}_{z i}=\left(\begin{array}{ccc}
0 & -S_{i} & X_{i} \\
S_{i} & 0 & Y_{i} \\
X_{i} & Y_{i} & 2 Z_{i}
\end{array}\right)(10)
$$

and $X_{i}=u_{0 i x} V_{i}, Y_{i}=u_{0 i y} V_{i}$ and $Z_{i}=u_{0 i z} V_{i}$

For small changes in $\boldsymbol{R}\left(q_{i}, \boldsymbol{u}_{0 i}\right)$, the hand orientation, Eq. (5), becomes

$$
\boldsymbol{R}_{\boldsymbol{h}}+\delta \boldsymbol{R}_{\boldsymbol{h}}=\prod_{i=1}^{n}\left[\boldsymbol{R}\left(q_{i}, \boldsymbol{u}_{0 i}\right)+\delta \boldsymbol{R}\left(q_{i}, \boldsymbol{u}_{0 i}\right)\right]
$$

Ignoring second and higher orders of variations, the above equation leads to

$$
\delta \boldsymbol{R}_{h} \boldsymbol{R}_{h}^{T}=\sum_{i=1}^{n}\left[\boldsymbol{R}_{i-1} \delta \boldsymbol{R}\left(q_{i}, \boldsymbol{u}_{0 i}\right) \boldsymbol{R}_{i}^{T}\right]
$$

The left-hand side of Eq. (12) is a skew symmetric matrix. It has only three significant elements namely $(3,2),(1,3)$ and $(2,1)$ or $\delta r_{1}, \delta r_{2}$ and $\delta r_{3}$. Therefore, $\delta \boldsymbol{R}_{h} \boldsymbol{R}_{h}^{T}$ could be converted to a vector $\delta \boldsymbol{r}_{h}$, which shows the errors in the endeffector orientation. Substituting $\delta R\left(q_{i}, u_{0 i}\right)$ from Eq. (9) into Eq. (12) yields

$$
\delta r_{h}=\sum_{i=I}^{n}\left\{\boldsymbol{R}_{i-I}\left(\boldsymbol{R}_{x i} \delta u_{0 i, x}+\boldsymbol{R}_{y i} \delta u_{0 i, y}+\boldsymbol{R}_{z i} \delta u_{0 i, z}\right) \boldsymbol{R}_{i}^{T}\right\}
$$

\section{POSITIONAL ERROR MODEL}

The position vector $P_{p}$ of a reference point $\mathrm{p}$ at the hand 


$$
\boldsymbol{P}_{p}=\sum_{i=1}^{n} b_{i+1}=\sum_{i=1}^{n} \boldsymbol{R}_{i} \boldsymbol{b}_{0, i+1}=\sum_{i=1}^{n}\left\{\left(\prod_{j=1}^{i} R\left(q_{j}, u_{0 j}\right)\right) b_{0, i+1}\right\}
$$

For small changes in $\boldsymbol{P}_{p}$, the position vector becomes

$$
\boldsymbol{P}_{p}+\delta \boldsymbol{P}_{p}=\sum_{i=1}^{n}\left\{\left(\prod_{j=I}^{i}\left(\boldsymbol{R}\left(q_{j}, u_{0 j}\right)+\delta \boldsymbol{R}\left(q_{j}, u_{0 j}\right)\right)\right)\left[\boldsymbol{b}_{0, i+1}+\delta \boldsymbol{b}_{0, i+1}\right]\right\}
$$

Ignoring second and higher orders of variations, the above equation leads to

$$
\delta \boldsymbol{P}_{p}=\sum_{i=1}^{n}\left\{\boldsymbol{R}_{i-1} \delta R\left(q_{i}, u_{0 i}\right)\left\{b_{0, i+1}+\sum_{j=i+1}^{n}\left[\left(\prod_{k=i+1}^{j} R\left(q_{k}, u_{0 k}\right)\right) b_{0, j+1}\right]\right\}\right\}+\sum_{i=1}^{n} \boldsymbol{R}_{i} \delta b_{0, i+1}(
$$

The above equation shows the errors in the end-effector position. Substituting $\delta R\left(q_{i}, u_{0 i}\right)$ from Eq. (9), the above equation becomes

$$
\delta \boldsymbol{P}_{p}=\sum_{i=l}^{n}\left\{\boldsymbol{R}_{i-I}\left(\boldsymbol{R}_{x i} \delta u_{0 i, x}+\boldsymbol{R}_{y i} \delta u_{o i, y}+\boldsymbol{R}_{z i} \delta u_{o i, z}\right) \boldsymbol{E}\right\}^{n} \sum_{i=l}^{n} \boldsymbol{R}_{i} \delta \boldsymbol{b}_{0, i+I}
$$

where

$$
\boldsymbol{E}=\boldsymbol{b}_{0, i+1}+\sum_{j=i+1}^{n}\left(\boldsymbol{R}\left(q_{j}, \boldsymbol{u}_{0 j}\right) \boldsymbol{b}_{0, j+1}\right)
$$

In order to insure the length constraint of the unit vectors, the following constraint is to be satisfied,

$$
\left\|u_{O i}\right\|=\sqrt{u_{O i, x}^{2}+u_{O i, y}^{2}+u_{O i, z}^{2}}=1
$$

For small changes,

$$
u_{0 i, x} \delta u_{0 i, x}+u_{0 i, y} \delta u_{0 i, y}+u_{0 i, z} \delta u_{0 i, z}=0 \quad \text { or } \quad u_{0 i}^{r} \delta u_{0 i}=0
$$

The above constraint is also implemented as an extension to the error Jacobian matrix.

\section{ERROR JACOBIAN MATRIX}

From Eqs. $(13,17,20)$, the following equation can be constructed,

$$
\left\{\begin{array}{l}
\delta \boldsymbol{u}_{0} \\
\delta b_{0}
\end{array}\right\}=\left\{\begin{array}{l}
\delta r_{h} \\
\delta p_{p}
\end{array}\right\}
$$


The above equation describes a linear relationship between the errors in the robot kinematic parameter elements $\left(\delta \boldsymbol{u}_{0 i}\right.$ and $\left.\delta \boldsymbol{b}_{0 i}\right)$, which can be defined as follows

$$
\begin{aligned}
& \delta u_{o}=\left(\begin{array}{lllll}
\delta u_{01 x} & \delta u_{01 y} & \delta u_{01 z} & \cdots & \delta u_{0 n z}
\end{array}\right)^{T} \\
& \delta b_{o}=\left(\begin{array}{lllll}
\delta b_{01 x} & \delta b_{01 y} & \delta b_{01 z} & \cdots & \delta b_{0 n z}
\end{array}\right)^{T}
\end{aligned}
$$

and the error in the position and orientation of the end-effector $\left(\delta r_{h}\right.$ and $\left.\delta p_{p}\right)$. The matrix $J$ represents the error Jacobian matrix, which can be expressed as

$$
J=\left(\begin{array}{ll}
J 1 & J 2 \\
J 3 & J 4 \\
J 5 & J 6
\end{array}\right)
$$

The $\mathrm{i}^{\text {th }}$ elements of the submatrix $\boldsymbol{J} \boldsymbol{I}$ are defined as

$$
J I_{i}=\left[\begin{array}{lll}
R_{i-I} R_{x i} R_{i}^{T} & R_{i-l} R_{y i} R_{i}^{T} \quad R_{i-I} R_{z i} R_{i}^{T}
\end{array}\right]
$$

while $\boldsymbol{J} \boldsymbol{2}_{\boldsymbol{i}}=\boldsymbol{0}$; and the $\mathrm{i}^{\text {th }}$ elements of $\boldsymbol{J}$ are

$$
J 3_{i}=\left\lfloor\begin{array}{lll}
R_{i-l} R_{x i} E & R_{i-l} R_{y i} E & R_{i-l} R_{z i} E
\end{array}\right]
$$

where $E$ is given by Eq. (18); and

$$
J 4_{i}=R_{i}, \quad J 5_{i}=u_{i} \quad \text { and } \quad J \sigma_{i}=0
$$

$J I$ to $J 4$ are $3 \times 6 n$; while $J 5$ and $J 6$ are $\mathrm{n} \times 6 \mathrm{n}$. Therefore, The error matrix $J$ is then $(6+n) \times 6 n$. The errors in the kinematic parameter elements $\left(\delta u_{0}\right.$ and $\left.\delta \boldsymbol{b}_{0}\right)$.

\section{CALIBRATION ALGORITHM}

In the calibration algorithm, the kinematic parameter errors are used to represent the GA, population and their Spectral norms represent the $\mathrm{GA}_{\mathrm{s}}$ cost functions. The algorithm could be proposed as follows:

1. The nominal link parameters $\left(u_{0 \mathrm{i}}\right.$ and $\left.\boldsymbol{b}_{0 \mathrm{i}}\right)$ and the joint variables $q$ are used for an arbitrary configuration.

2. The nominal hand orientation and position $\boldsymbol{R}_{\mathrm{h}}$ and $\boldsymbol{P}_{\mathrm{p}}$ are computed by using Eqs. $(5,14)$.

3. The error Jacobian matrix $J$ is constructed by using Eqs. $(25-27)$. 
4. The actual joint variables $\boldsymbol{q}_{a}$ are calculated by adding a range of random error values.

5. The GA iterations is started by generating some initial populations for the link parameter errors $\delta \boldsymbol{u}_{0 i}$ and $\delta \boldsymbol{b}_{0 i}$. These populations are used as parents from which the genetic operators are applied to produce new offspring population.

6. The actual hand orientation and position $\boldsymbol{R}_{h}^{a}$ and $\boldsymbol{P}_{p}^{a}$ are computed as in step 2 by utilizing the actual joint variables.

7. The right hand side of Eq. (21) is obtained as

$$
\delta \boldsymbol{r}=\delta \boldsymbol{R}_{k} \boldsymbol{R}_{k}^{T} \text { and } \delta \boldsymbol{P}_{p}=\boldsymbol{P}_{p}^{a}-\boldsymbol{P}_{p}
$$

where

$$
\delta \boldsymbol{R}_{h}=\boldsymbol{R}_{h}^{a}-\boldsymbol{R}_{h}
$$

8. The offspring populations are computed from the following relationship (the Pseudo inverse is used).

$$
\left\{\begin{array}{l}
\delta \boldsymbol{u}_{0} \\
\delta \boldsymbol{b}_{0}
\end{array}\right\}=J^{-1}\left\{\begin{array}{l}
\delta \boldsymbol{r}_{\boldsymbol{h}} \\
\delta \boldsymbol{p}_{p}
\end{array}\right\}
$$

Then, their population costs are evaluated by computing their Spectral norms.

9. The offspring together with their parents are evaluated by their costs. The most fit population are those with the lowest costs.

10. This process is iterated until a certain criterion (such as a certain number of iterations) is met.

\section{A CASE STUDY}

A Numerical example is presented in an arbitrary configuration for a six-degrees of freedom PUMA-type manipulator (Fig. 1). Its joint variables are chosen as

$$
q=\left(\begin{array}{llllll}
-2.741 & 4.501 & 2.609 & 2.044 & 0.389 & 2.285
\end{array}\right)^{T}
$$

The nominal kinematic parameters are listed in Table 1. The joint value errors are randomly taken from a range of \pm 0.001 radians; while the kinematic parameter errors are randomly taken from a range of \pm 0.01 for screw axes $\boldsymbol{u}^{\text {ss }}$ and \pm 0.5 for body vectors $b^{\text {ss }}$. 


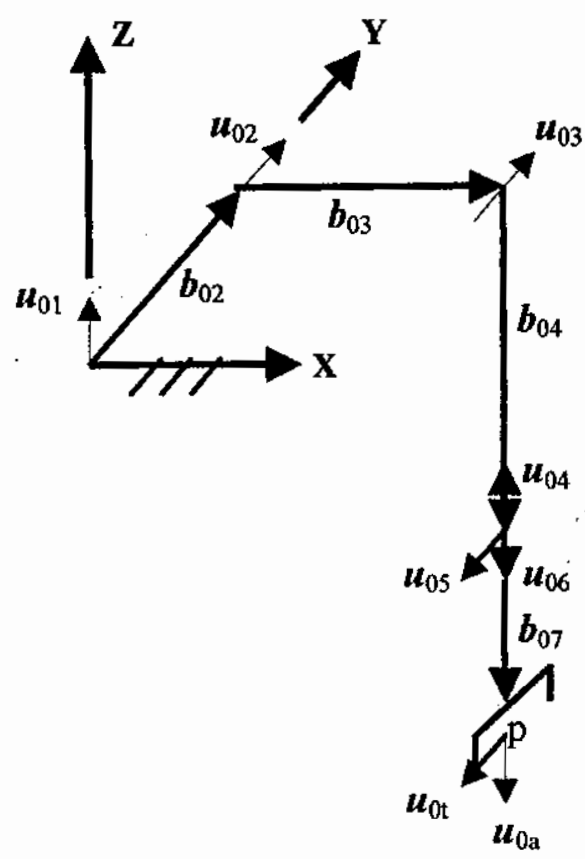

Fig. 1. PUMA-type robot in its ZRP configuration

Table 1. Nominal kinematic parameters

\begin{tabular}{|c|c|c|c|c|c|c|c|c|c|c|c|}
\hline \multicolumn{6}{|c|}{$\boldsymbol{u}_{01}: \boldsymbol{u}_{06}$} & \multicolumn{6}{|c|}{$\boldsymbol{b}_{02}: \boldsymbol{b}_{07}(\mathrm{~mm})$} \\
\hline 0 & 0 & 0 & 0 & 0 & 0 & $\overline{0}$ & 500 & 0 & 0 & $\overline{0}$ & 0 \\
\hline 0 & 1 & 1 & 0 & -1 & 0 & 200 & $v$ & 0 & 0 & 0 & 0 \\
\hline & 0 & 0 & 1 & 0 & & 0 & 0 & -500 & 0 & 0 & -100 \\
\hline
\end{tabular}

Initial populations are generated to represent the parents from which an initial set of errors along with their initial cost are computed and listed in Table 2. The $\mathrm{GA}^{\text {ss }}$ operators are then applied to generate the offspring. This algorithm is converged after 11 iterations. The optimal kinematic parameter errors and their cost are given in Table 3. Whereas, the algorithm convergence are shown in Fig. 2 .

Table 2. Initial population with its cost

\begin{tabular}{|c|c|c|c|c|c|c|c|c|c|c|c|c|}
\hline \multicolumn{6}{|c|}{$\delta u_{01}: \delta u_{06}\left(\times 10^{-2}\right)$} & \multicolumn{6}{|c|}{$\delta b_{02}: \delta b_{07}(\mathrm{~mm})$} & Cost \\
\hline-0.29 & 0.64 & 0.57 & -0.12 & 0.95 & -0.29 & 0.15 & 0.09 & -0.26 & 0.23 & 0.31 & -0.48 & \\
\hline 0.76 & -0.51 & 0.53 & 0.00 & -0.99 & 0.77 & -0.29 & -0.07 & -0.37 & 0.27 & -0.47 & -0.08 & 0.0112 \\
\hline 0.01 & 0.63 & 0.25 & 0.92 & 0.77 & -0.26 & -0.09 & -0.47 & -0.07 & -0.36 & 0.16 & -0.00 & \\
\hline
\end{tabular}

Table 3. Final optimal population with its cost

\begin{tabular}{|c|c|c|c|c|c|c|c|c|c|c|c|c|}
\hline \multicolumn{6}{|c|}{$\delta u_{01}: \delta u_{06}\left(\times 10^{-2}\right)$} & \multicolumn{6}{|c|}{$\delta b_{02}: \delta b_{07}(\mathrm{~mm})$} & Cost \\
\hline-0.29 & 0.64 & 0.77 & -0.99 & 0.00 & 0.53 & -0.30 & -0.32 & -0.07 & -0.44 & -0.33 & -0.30 & \\
\hline 0.76 & -0.51 & -0.29 & 0.95 & -0.12 & 0. & -0 & 0.20 & -0.38 & -0.47 & -0.38 & -0.49 & 0.0027 \\
\hline 0.01 & 0.92 & 0.77 & 0.63 & 0.25 & -0.26 & -0.46 & 0.11 & -0.19 & -0.40 & -0.27 & -0.48 & \\
\hline
\end{tabular}

From the foregoing example, it has been shown that the initial cost is 0.0112 and the final optimal cost is 0.0027 . Therefore, the optimal kinematic parameter errors are reduced by $24.1 \%$. 


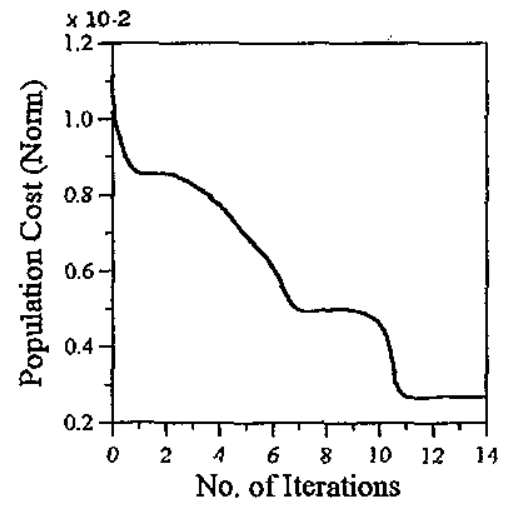

Fig. 2. Convergence of the algorithm

\section{CONCLUSIONS}

The Genetic Algorithm procedure is used to calibrate the robot kinematic errors based on the zero position analysis method. The effectiveness of the algorithm and its convergence in the presence of small joint errors and measurement errors is demonstrated through a numerical experiment. The kinematic parameter errors are reduced the by $24.1 \%$.

\section{REFERENCES}

[1] Goldberg, D.E., "Genetic Algorithms in Search, Optimization, and Machine Learning", Addison-Wesley, 1989

[2] Zhuang, H., Wu, J. and Huang. W., "Optimal planning of Robot Calibration Experiments by Genetic Algorithms", Journal of Robotic Systems, Vol. 14 No. 10, John Wiley \& Sons Inc, 1997.

[3] Maturana, F., Gu, P., Naumann, A. and Norrie, D.H., "Object-Oriented Job-Shop Scheduling Using Genetic Algorithms", Computers in Industry, Vol. 32, No. 3, pp. 281-294, 1997.

[4] Bozer, Y.A., Schorn, E.C. and Sharp, G.P., "Geometric Aproaches to solve the Chebyshev Traveling Salesman Problem", IIE Transactions, Vol. 22, pp. 238-254, 1990.

[5] Samak, S., "Optimal Component Assembly Planning of Printed Circuit Using Genetic Algorithm", $2^{\text {nd }}$ Assiut University Inter. Conf. on Mechanical Engineering Advanced Technology for Industrial Production, pp. 377-380, Assiut, Egypt, 1999.

[6] Veitschegger, W.K. and Wu, C., "Robot Accuracy Analysis Based on Kinematics", IEEE Journal of Robotics and Automation, Vol. RA-2, No. 3, pp. 171-179, 1986.

[7] Wu, C., "The Kinematic Error Model for the Design of Robot Manipulators", American Control Conference, pp.497-502, San Francisco, 1983.

[8] Vukobratović, M. and Borovac, B., "Accuracy of the Robot Positioning and Orientation Assessed Via its Manufacturing Tolerances", Mechanism and Machine Theory, Vol. 30, No. 1, pp. 11-32, 1995. 
[9] Bruyninckx, H., Demey, S., Dutré, S. and Schutter, J.D., "Kinematic Models for Model-Based Compliant Motion in the Presence of Uncertainty", The International Journal of Robotics Research, Vol. 14, No. 5, pp. 465-482, 1995.

[10] Samak, S., El-Sayed, S. El-Shakery, S. and El-Dardierie, M., Kinematic Perturbations on Robot Precision", $7^{\text {th }}$ IFToMM International Symposium on the Theory of Machines and Mechanisms, pp. 327-332, Bucharest, Romania, 1997.

[11] Kazerounian, K. and Qian, G.Z., "Kinematic Calibration of Robotic Manipulators", ASME Journal of Mechanisms, Transmissions, and Automation in Design, Vol. 111, pp.482-487, 1989.

[12] Gupta, K.C., "A Note on Position Analysis of Manipulators", Mechanism and Machine Theory, Vol. 19, pp. 5-8, 1984.

[13] Samak, S. and Gupta, K.C., "Parametric Uncertainty on Manipulators Dynamics", Mechanism and Machine Theory, Vol. 33, No. 7, pp. 945-956, 1998.

[14] Gupta, K.C., "Mechanics and Control of Robots", Springer, New York, 1997. 
دقة المعايرة الكينماتيكية للإنسان الآلى باستخدام طريقة الجينات المواثية

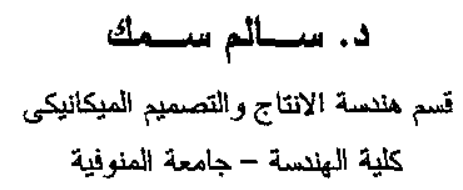

ملنص البحث:

نتأئز دقة وضع ودوران النهاية الطرفية للإنسان الآلى بدقة قيم عناصره الكينماتيكية، وهذا الشرط من الصعب نوافره فى الحياة العملية، لذا فلابد من إسيتثباط تثنيات

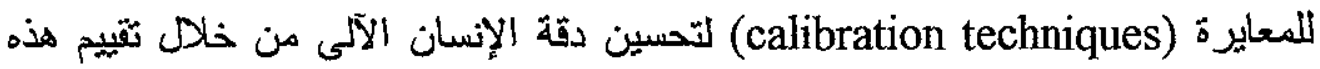
القيم.

ومن ثم فإن هذا البحث يقام طريقة جليدة لمعايرة الدقة الكينماتيكية للإنسان الآلى

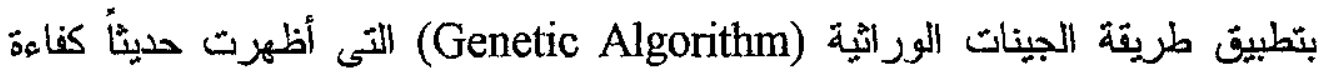

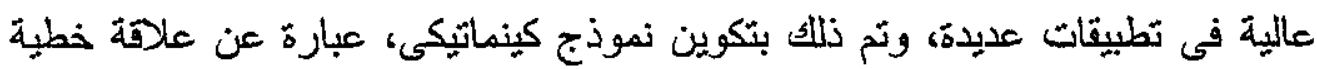

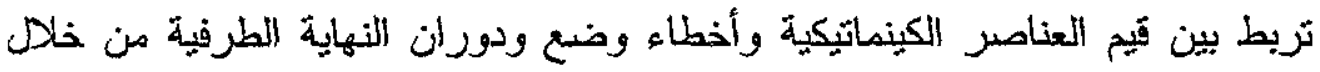

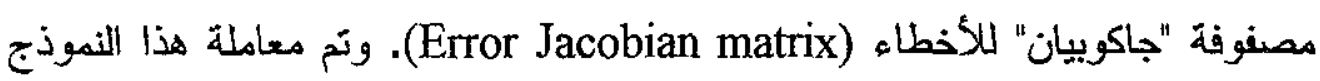
كمُكلة هنالية (optimization problem) لمناو لات الإنسان الآلى.

والهدف من ذلك هو تمليل وتقيبم أداء طريقة الجينات. الوراثية فى معايرة أخطاء الإنسان الآلى، ففى هذه الطريقة أخطاء بارامثزات الإنسان الآلى تمثل مجتمع الآباء والأبناء، ومعيار مصفوفة الأخطاء تمثل دالة التكاليف (cost function).

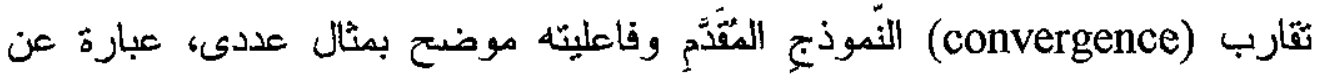

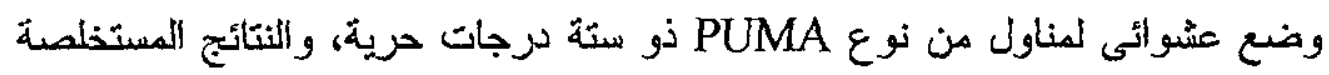

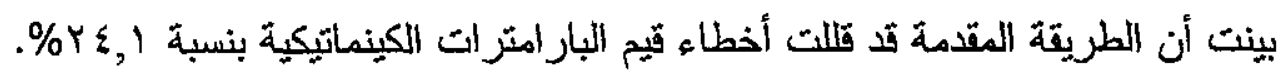

